\title{
Minimum bias multiple taper spectral estimation *
}

\author{
Kurt S. Riedel and Alexander Sidorenko \\ Courant Institute of Mathematical Sciences, New York University \\ New York, New York 10012-1185
}

EDICS: SP 3.1.1

\begin{abstract}
Two families of orthonormal tapers are proposed for multitaper spectral analysis: minimum bias tapers, and sinusoidal tapers $\left\{\mathbf{v}^{(k)}\right\}$, where $v_{n}^{(k)}=\sqrt{\frac{2}{N+1}} \sin \frac{\pi k n}{N+1}$, and $N$ is the number of points. The resulting sinusoidal multitaper spectral estimate is $\hat{S}(f)=\frac{1}{2 K(N+1)} \sum_{j=1}^{K} \mid y(f+$ $\left.\frac{j}{2 N+2}\right)-\left.y\left(f-\frac{j}{2 N+2}\right)\right|^{2}$, where $y(f)$ is the Fourier transform of the stationary time series, $S(f)$ is the spectral density, and $K$ is the number of tapers. For fixed $j$, the sinusoidal tapers converge to the minimum bias tapers like $1 / N$. Since the sinusoidal tapers have analytic expressions, no numerical eigenvalue decomposition is necessary. Both the minimum bias and sinusoidal tapers have no additional parameter for the spectral bandwidth. The bandwidth of the $j$ th taper is simply $\frac{1}{N}$ centered about the frequencies $\frac{ \pm j}{2 N+2}$. Thus the bandwidth of the multitaper spectral estimate can be adjusted locally by simply adding or deleting tapers. The band limited spectral concentration, $\int_{-w}^{w}|V(f)|^{2} d f$, of both the minimum bias and sinusoidal tapers is very close to the optimal concentration achieved by the Slepian tapers. In contrast, the Slepian tapers can have the local bias, $\int_{-1 / 2}^{1 / 2} f^{2}|V(f)|^{2} d f$, much larger than of the minimum bias tapers and the sinusoidal tapers.
\end{abstract}

*The authors thank D. J. Thomson and the referees for useful comments. Research funded by the U.S. Department of Energy. 


\section{Introduction}

We consider a stationary time series, $\left\{x_{n}, n=1 \ldots N\right\}$ with a spectral density, $S(f)$. A common estimator of the spectral density is to smooth the square of the discrete Fourier transform (DFT) locally:

$$
\hat{S}(f)=\frac{1}{(2 L+1) N} \sum_{j=-L}^{L}\left|y\left(f+\frac{j}{N}\right)\right|^{2},
$$

where $y(f)$ is the Fourier transform (FT) of the stationary time series: $y(f) \equiv \sum_{n=1}^{N} x_{n} e^{-i 2 \pi n f}$. Since (1) is quadratic in the FT, $y(f)$, it is natural to consider a more general class of quadratic spectral estimators. We examine quadratic estimators where the underlying self-adjoint matrix has rank $K$, where $K$ is prescribed. Using the eigenvector representation, the resulting quadratic spectral estimator can be recast as a weighted sum of $K$ orthonormal rank one spectral estimators. This class of spectral estimators was originally proposed by Thomson [19] under the name of multiple taper spectral analysis (MTSA). We refer the reader to [3, 8, 11, 12, 16, 18, 19] for excellent expositions and generalizations of Thomson's theory.

In MTSA, a rank $K$ quadratic spectral estimate is constructed by choosing an orthonormal family of tapers/spectral windows and then averaging the $K$ estimates of the spectral density. In practice, only the Slepian tapers (also known as discrete prolate spheroidal sequences [17]) are routinely used for MTSA.

In the present paper, we propose and analyze two new orthonormal families of tapers: minimum bias (MB) tapers and sinusoidal tapers. The MB tapers minimize the local frequency bias, $\int f^{2}|V(f)|^{2} d f$, subject to orthonormality constraints, where $V(f)$ is the DFT of the taper. For continuous time, the MB tapers have simple analytic expressions. The first taper in the family is Papoulis' optimal taper [9]. For discrete time, the MB tapers satisfy a selfadjoint eigenvalue problem and may be computed numerically.

In the case of discrete time, we define the $k$ th sinusoidal taper, $\mathbf{v}^{(k)}$, as $v_{n}^{(k)}=\sqrt{\frac{2}{N+1}} \sin \frac{\pi k n}{N+1}$, where $N$ is the sequence length. The sinusoidal tapers are an orthonormal family that converge to the MB tapers with rate $1 / N$ as $N \rightarrow \infty$. These results are given in Section 3. Section 4 compares the local bias, $\int_{-1 / 2}^{1 / 2} f^{2}|V(f)|^{2} d f$, and the spectral concentration, $\int_{-w}^{w}|V(f)|^{2} d f$, of the MB tapers, the sinusoidal tapers and the Slepian tapers. 
In Section 5, we show that the quadratic spectral estimator which minimizes the expected square local error is weighted multitaper estimate using the MB tapers. A local error analysis is given and the optimal number of tapers is determined. At frequencies where the spectral density is changing rapidly, fewer tapers should be used. In Section 6, we show that kernel smoother spectral estimates [4, 10] are multitaper estimates and we show that smoothing the logarithm of the multitaper estimate significantly reduces the variance in comparison with smoothing athe logarithm of a single taper estimate. We also describe our data adaptive method for estimating the spectrum. In Section 7, we apply our spectral estimation techniques to real data and show that our tapers outperform the Slepian tapers whenever a variable bandwidth is needed. In the Appendix, we show that the leading principal components of kernel smoother spectral estimates resemble the MB tapers.

\section{Quadratic Estimators of the Power Spec- trum}

Let $N$ discrete measurements, $x_{1}, x_{2}, \ldots, x_{N}$, be given as a realization of a stationary stochastic process. We normalize the time interval between measurements to unity. The Cramer representation of a discrete stationary stochastic process [4, 12] is

$$
x(t)=\int_{-1 / 2}^{1 / 2} e^{2 \pi i n f} d Z(f),
$$

where $d Z$ has independent spectral increments: $E[d Z(f) d \bar{Z}(g)]=S(f) \delta(f-$ $g) d f d g$. We assume that the spectral density, $S(f)$, is twice continuously differentiable.

The spectral inverse problem is to estimate the spectral density, $S(f)$, given $\left\{x_{n}\right\}$. As shown in [3, 8], every quadratic, modulation-invariant power spectrum estimator has the form:

$$
\widehat{S}(f)=\sum_{n, m=1}^{N} q_{n m} e^{2 \pi i(m-n) f} x_{n} x_{m},
$$

where $\mathbf{Q}=\left[q_{n m}\right]$ is a symmetric matrix of order $N$ and does not depend on frequency. Consider the eigenvector decomposition: $\mathbf{Q}=\sum_{k=1}^{K} \mu_{k} \mathbf{v}^{(k)}\left(\mathbf{v}^{(k)}\right)^{T}$, 
where $K$ is the rank of $\mathbf{Q}$, and $\mathbf{v}^{(1)}, \mathbf{v}^{(2)}, \ldots, \mathbf{v}^{(K)}$ is an orthogonal system of eigenvectors. The multitaper representation of the quadratic spectral estimator is

$$
\widehat{S}(f)=\sum_{k=1}^{K} \mu_{k}\left|\sum_{n=1}^{N} v_{n}^{(k)} x_{n} e^{-2 \pi i n f}\right|^{2} .
$$

In the case $K=1$, estimator (3) turns out a tapered periodogram estimator:

$$
\widehat{S}_{v}(f)=\left|\sum_{n=1}^{N} v_{n} x_{n} e^{-2 \pi i n f}\right|^{2}
$$

with a taper $\mathbf{v}=\left(v_{1}, v_{2}, \ldots, v_{N}\right)^{T}$. If the tapering is uniform (i.e. $v_{1}=v_{2}=$ $\left.\ldots=v_{N}=\frac{1}{\sqrt{N}}\right)$, we name (4) the periodogram estimator. The estimator (3) is a linear combination of $K$ orthogonal tapered periodogram estimators. In MTSA, $K$ is normally chosen to be much less than $N$. The multiple taper spectral estimate can be thought of as a low rank, "principal components" approximation of a general quadratic estimator. Multiple taper analysis has also been applied to nonstationary spectral analysis [13, 1].

In practice, one does not begin the analysis with a given quadratic estimator, Q. Instead, one usually specifies a family of orthonormal tapers $\left\{\mathbf{v}^{(1)}, \ldots, \mathbf{v}^{(K)}\right\}$ with desirable properties. Previously, only the family of Slepian tapers were used in practice. The goal of this article is to introduce other families of tapers.

We define the $k$ th spectral window, $V^{(k)}$, to be the FT of the $k$ th taper:

$$
V^{(k)}(f)=\sum_{n=1}^{N} v_{n}^{(k)} e^{-i 2 \pi n f} .
$$

The tapers are normally chosen to have their spectral density localized near zero frequency. We define two common measures of frequency localization.

The local bias of a spectral window $V$ is $\int_{-1 / 2}^{1 / 2} f^{2}|V(f)|^{2} d f$. The term "local bias" is used because it is proportional to the leading order term in the bias error of a taper estimate as $N \rightarrow \infty$.

The spectral concentration in band $[-w, w]$ is defined as $\int_{-w}^{w}|V(f)|^{2} d f$. The bandwidth, $w$, is a free parameter. The Slepian tapers are the unique sequences which maximize the spectral concentration subject to the constraint that they form an orthonormal family. Detailed analysis of the Slepian sequences is given in [17]. We stress that the Slepian tapers depend on the 
bandwidth parameter, $w$, and that the first $2 N w$ spectral windows are concentrated in the band $[-w, w]$ while the remaining windows are concentrated outside.

\section{Minimum Bias Tapers}

\subsection{Continuous Time Case}

We consider time-limited signals; the time interval is normalized to $[0,1]$. In the time domain, the taper $\nu(t)$ is a function in $\mathcal{L}_{2}[0,1]$ which we normalize to $\int_{0}^{1} \nu^{2}(t) d t=1$. The functions $\{\sin (\pi k t), k=1,2, \ldots\}$ form a complete orthogonal basis on $[0,1]$. (Completeness can be proven by extending $\nu(t)$ to be an odd function on $[-1,1]$ and using the completeness of the complex exponentials on $[-1,1]$. See [5].)

Setting $a_{k}=2 \int_{0}^{1} \nu(t) \sin (\pi k t) d t$, then $\sum_{k=1}^{K} a_{k} \sin (\pi k t) d t$ converges to $\nu(t)$ in $\mathcal{L}_{2}[0,1]$ as $K \rightarrow \infty$. The taper normalization is equivalent to $\frac{1}{2} \sum_{k=1}^{\infty} a_{k}^{2}=$ 1. The Fourier transform of the taper is the complex-valued, spectral window function:

$$
V(f)=\int_{0}^{1} \nu(t) e^{-i 2 \pi f t} d t .
$$

$V(f)$ is defined on the frequency domain $[-\infty, \infty]$, belongs to $\mathcal{L}_{2}[-\infty, \infty]$, and satisfies $\int_{-\infty}^{\infty}|V(f)|^{2} d f=2 \pi \int_{0}^{1} \nu^{2}(t) d t=2 \pi$.

The local bias of a taper spectral estimate [4, 9, 10] is

$E[\widehat{S}(f)]-S(f)=\int_{-\infty}^{\infty}|V(g-f)|^{2}(S(g)-S(f)) d g \approx \frac{S^{\prime \prime}(f)}{2} \int_{-\infty}^{\infty}|V(h)|^{2} h^{2} d h$.

We consider tapers which minimize the leading order term:

$$
\begin{aligned}
\int_{-\infty}^{\infty}|V(f)|^{2} f^{2} d f & =\int_{0}^{1}\left(\frac{1}{2 \pi} \frac{d}{d t} \sum_{k=1}^{\infty} a_{k} \sin (\pi k t)\right)^{2} d t \\
& =\frac{1}{4} \int_{0}^{1}\left(\sum_{k=1}^{\infty} a_{k} k \sin (\pi k t)\right)^{2} d t=\frac{1}{8} \sum_{k=1}^{\infty} a_{k}^{2} k^{2} .
\end{aligned}
$$

The last expression attains the global minimum when $a_{1}^{2}=2, a_{2}=a_{3}=$ $\ldots=0$. Hence, the leading order term in the bias expression (7) is minimal for the taper $\sqrt{2} \sin (\pi t)$ (This result was obtained by Papoulis [9]). In [9], Papoulis extends $\nu(t)$ to be zero outside of $(0,1)$, and therefore has a Fourier 
integral representation of $\nu(t)$. We have extended $\nu(t)$ to be periodic and vanish at each integer value. Since $\nu(t)$ is optimized for $t \in[0,1]$, both representations are valid.

Equation (8) implies the more general result:

Theorem $3.1 v_{k}(t)=\sqrt{2} \sin (\pi k t)(k=1,2, \ldots)$ is the only system of functions in $\mathcal{L}_{2}[0,1]$ which satisfy the requirements:

(i) $\int_{0}^{1} v_{k}^{2}(t) d t=1$, and

(ii) $\quad v_{k}$ minimizes $\int_{-\infty}^{\infty}\left|V^{(k)}(f)\right|^{2} f^{2} d f$ in the subspace of func-

tions orthogonal to $v_{1}, \ldots, v_{k-1}$.

The kth minimum value is $\int_{-\infty}^{\infty}\left|V^{(k)}(f)\right|^{2} f^{2} d f=\frac{k^{2}}{4}$.

We name $v_{k}(t)=\sqrt{2} \sin (\pi k t)(k=1,2, \ldots)$ the continuous time minimum bias tapers. The Fourier transform of $v_{k}(t)$ is

$$
\begin{aligned}
V^{(k)}(f) & =\frac{e^{-i \pi\left(f-\frac{k}{2}\right)}}{i \sqrt{2}}\left\{\frac{\sin \left[\pi\left(f-\frac{k}{2}\right)\right]}{\pi\left(f-\frac{k}{2}\right)}-(-1)^{k} \frac{\sin \left[\pi\left(f+\frac{k}{2}\right)\right]}{\pi\left(f+\frac{k}{2}\right)}\right\} \\
& =e^{-i \pi\left(f-\frac{k-1}{2}\right)} \cdot \frac{k^{2} \sin \left(\pi f-\frac{\pi k}{2}\right)}{4 \sqrt{2} \pi\left[f^{2}-\left(\frac{k}{2}\right)^{2}\right]}
\end{aligned}
$$

Thus, $\left|V^{(k)}(f)\right|$ decays as $f^{-2}$ for large frequencies.

\subsection{Discrete Time Case}

We now consider the discrete time domain $\{1,2, \ldots, N\}$ with the corresponding normalized frequency domain $\left[-\frac{1}{2}, \frac{1}{2}\right]$. A taper is a vector, $\boldsymbol{\nu}=$ $\left(\nu_{1}, \ldots, \nu_{N}\right)$, normalized by $\sum_{n=1}^{N}\left(\nu_{n}\right)^{2}=1$. By the same argument as in the previous section, the leading order term of the bias of a taper spectral estimate is proportional to the local bias, $\int_{-\frac{1}{2}}^{\frac{1}{2}}|V(f)|^{2} f^{2} d f$, where the frequency window, $V(f)$, is defined in Eq. (5).

Lemma 3.2 For the frequency window of a discrete time taper,

$$
\int_{-\frac{1}{2}}^{\frac{1}{2}}|V(f)|^{2} f^{2} d f=\boldsymbol{\nu} \mathbf{A} \nu^{*}
$$


where $\mathbf{A}=\left[a_{n m}\right]$ with

$$
a_{n m}=\int_{-\frac{1}{2}}^{\frac{1}{2}} e^{i 2 \pi(n-m) f} f^{2} d f=\left\{\begin{array}{l}
\frac{1}{12} \quad \text { if } n=m \\
\frac{(-1)^{n-m}}{2 \pi^{2}(n-m)^{2}} \text { if } n \neq m .
\end{array}\right.
$$

Corollary 3.3 The tapers $\boldsymbol{\nu}^{(1)}, \boldsymbol{\nu}^{(2)}, \ldots, \boldsymbol{\nu}^{(N)}$, defined by the requirements

(i) $\sum_{n=1}^{N}\left(\nu_{n}^{(k)}\right)^{2}=1$

(ii) $\boldsymbol{\nu}^{(k)}$ minimizes $\int_{-\frac{1}{2}}^{\frac{1}{2}}\left|V^{(k)}(f)\right|^{2} f^{2} d f$ in the subspace of vectors orthogonal to $\boldsymbol{\nu}^{(1)}, \ldots, \boldsymbol{\nu}^{(k-1)}$,

are the eigenvectors of the matrix $\mathbf{A}$ sorted in the increasing order of the eigenvalues. The integral $\int_{-\frac{1}{2}}^{\frac{1}{2}}\left|V^{(k)}(f)\right|^{2} f^{2} d f$ is equal to the kth eigenvalue.

We name $\boldsymbol{\nu}^{(1)}, \boldsymbol{\nu}^{(2)}, \ldots, \boldsymbol{\nu}^{(N)}$ the discrete minimum bias tapers. They can be approximated by the sinusoidal tapers, $\mathbf{v}^{(1)}, \mathbf{v}^{(2)}, \ldots, \mathbf{v}^{(N)}$, which are discrete analogs of the continuous time minimum bias tapers. Namely, we define $\mathbf{v}^{(k)}=\left(v_{1}^{(k)}, \ldots, v_{N}^{(k)}\right)^{T}$ with $v_{n}^{(k)}=\sqrt{\frac{2}{N+1}} \sin \frac{\pi k n}{N+1}, k=1,2, \ldots, N$.

Lemma 3.4 The sinusoidal tapers, $\mathbf{v}^{(1)}, \mathbf{v}^{(2)}, \ldots, \mathbf{v}^{(N)}$, form an orthonormal basis in $\mathbf{R}^{N}$ and the local bias of $\mathbf{v}^{(k)}$ is $\frac{k^{2}}{4 N^{2}}\left(1+\mathcal{O}\left(\frac{1}{N}\right)\right)$.

Corollary 3.5 The multitaper estimate (3) using $K$ sinusoidal tapers has local bias equal to $\sum_{k=1}^{K} \mu_{k} \frac{k^{2}}{4 N^{2}}+\mathcal{O}\left(\frac{K^{2}}{N^{3}}\right)$ and has the following representation:

$$
\hat{S}(f)=\sum_{j=1}^{K} \frac{\mu_{j}}{2(N+1)}\left|y\left(f+\frac{j}{2 N+2}\right)-y\left(f-\frac{j}{2 N+2}\right)\right|^{2} .
$$

The uniformly weighted estimate, $\mu_{k}=\frac{1}{K}$, has local bias equal to $\frac{K^{2}}{12 N^{2}}+$ $\mathcal{O}\left(\frac{K^{2}}{N^{3}}\right)$.

From (9), the reason for the low bias of the sinusoidal tapers is apparent: the frequency sidelobe from $y\left(f+\frac{j}{2 N+2}\right)$ cancels the sidelobe of $y\left(f-\frac{j}{2 N+2}\right)$. As a result, the sidelobe of $y\left(f+\frac{j}{2 N+2}\right)$ minus $y\left(f-\frac{j}{2 N+2}\right)$ is much smaller than that of the periodogram.

Our preferred weighting is the parabolic weighting: $\mu_{j}=C\left(1-j^{2} / K^{2}\right)$ because the parabolic weighting minimizes the expected square error in kernel smoothers as $K$ and $N$ tend to infinity. Since the weights decrease smoothly to zero, the resulting estimate is smooth in frequency. 
Corollary 3.6 The uniformly weighted multitaper estimate using $K$ sinusoidal tapers can be computed in $\mathcal{O}(N \ln N)+\mathcal{O}(K N)$ operations while the generic multitaper estimate requires $\mathcal{O}(K N \ln N)$ operations plus the cost of computing the $K$ tapers.

The following result demonstrates that the $k$ th spectral window is concentrated on $\left[\frac{k-1}{2(N+1)}, \frac{k+1}{2(N+1)}\right] \cup\left[-\frac{k+1}{2(N+1)},-\frac{k-1}{2(N+1)}\right]$.

Corollary 3.7 The Fourier transform of $\mathbf{v}^{(k)}$ equals

$$
\begin{aligned}
V^{(k)}(f) & =\frac{e^{-i \pi\left((N+1) f-\frac{k}{2}\right)}}{i \sqrt{2(N+1)}}\left\{\frac{\sin \left[N \pi\left(f-\frac{k}{2(N+1)}\right)\right]}{\sin \left[\pi\left(f-\frac{k}{2(N+1)}\right)\right]}-(-1)^{k} \frac{\sin \left[N \pi\left(f+\frac{k}{2(N+1)}\right)\right]}{\sin \left[\pi\left(f+\frac{k}{2(N+1)}\right)\right]}\right\} \\
& =e^{-i \pi\left((N+1) f-\frac{k-1}{2}\right)} \cdot \frac{\sin \frac{\pi k}{N+1}}{\sqrt{2(N+1)}} \cdot \frac{\sin \left[(N+1) \pi f-\frac{\pi k}{2}\right]}{\sin ^{2}(\pi f)-\sin ^{2} \frac{\pi k}{2(N+1)}} .
\end{aligned}
$$

Thus $\left|V^{(k)}(f)\right|=\sqrt{\frac{N+1}{2}}$ for $|f|=\frac{k}{2(N+1)}$, and $\left|V^{(k)}(f)\right| \sim \frac{1}{N^{3 / 2}}$ for $f=$ $\mathcal{O}(1)$. In particular, $\left|V^{(k)}(f)\right| \approx \frac{\pi k}{\sqrt{2} N^{3 / 2}}$ when $f \rightarrow \frac{1}{2}$. In the intermediate frequencies, $\frac{k}{2(N+1)}<f \ll \frac{1}{2},\left|V^{(k)}(f)\right|$ decays as $\frac{1}{f^{2}}$.

Numerical evaluation (see Table 1) shows that $\left\|\mathbf{v}^{(k)}-\boldsymbol{\nu}^{(k)}\right\|_{L_{2}}<\frac{k}{4(N+2)}$

for all $k$. The same rate of convergence is observed in $L_{\infty}$ norm: $\left\|\frac{\mathbf{v}^{(k)}}{\left\|\mathbf{v}^{(k)}\right\|_{L_{\infty}}}-\frac{\boldsymbol{\nu}^{(k)}}{\left\|\boldsymbol{\nu}^{(k)}\right\|_{L_{\infty}}}\right\|_{L_{\infty}}<$ $\frac{k}{2(N+2)} \cdot\left(\|\cdot\|_{L_{\infty}}\right.$ is the supremum norm in the time domain.) Figure 1 plots the envelopes of $\left|V^{(k)}(f)\right|^{2}$ for both the minimum bias and sinusoidal tapers with $N=200, k=1$. The spectral energy of both tapers is nearly identical for $|f|<.25$. Near the Nyquist frequency, the spectral energy of the sinusoidal taper is roughly three times larger than that of the minimum bias taper. In the time domain, the sinusoidal tapers are virtually indistinguishable from the MB tapers.

\section{Comparison of Spectral Localizations}

We now compare the local bias and the spectral concentration of three families of orthonormal tapers: minimum bias (MB) tapers, sinusoidal tapers and Slepian tapers. Both the local bias and the spectral concentration of the 
Slepian tapers depend on the bandwidth parameter, $w$. For properties of the Slepian tapers, we refer the reader to [12, 16, 17, 18, 19].

Since the MB tapers minimize the local bias, clearly the sinusoidal tapers and the Slepian tapers have larger local bias. The only question is whether the difference is large or small. Table 2 gives the local bias, $\sum_{k=1}^{K} \int_{-1 / 2}^{1 / 2} f^{2}\left|V^{(k)}(f)\right|^{2} d f$, of the three families of tapers for $N=50$. The sinusoidal tapers come within $0.2 \%$ of achieving the optimal local bias. In contrast, the local bias of the Slepian tapers can be many times larger.

We compute the local bias for three different values of the bandwidth, $w$. The general pattern is that the $k$ th Slepian taper has roughly the same local bias as the MB taper does when $N w<k<2 N w$. The ratio of the local bias of the Slepian tapers to that of the MB tapers is smallest at $k \approx 1.2 N w$. As $|k-1.2 N w|$ increases, the local bias rapidly departs from the optimal value.

Table 3 compares the spectral concentration of the tapers for $N=50$ and $w=.08$. Both the MB tapers and the sinusoidal tapers are within $1.7 \%$ of the optimal value, except for $k=8,9$. Notice that $2 N w=8$. Although the ratio of the spectral concentration, $\int_{-w}^{w}|V(f)|^{2} d f$, for the MB and sinusoidal tapers to that of the Slepian tapers is usually very close to one, the ratio of the spectral energy outside of the frequency band $|f|<w$ can be quite large. Thus our conclusions depend on using $\int_{-w}^{w}|V(f)|^{2} d f$ and not $1-\int_{-w}^{w}|V(f)|^{2} d f$ as the measure of frequency concentration.

Figures 1-3 compare $\left|V^{(k)}(f)\right|^{2}$ of the Slepian and MB tapers for $N=200$. For Figures 1 and 2, we select the Slepian parameter, $w$, equal to .01 so that $K=2 N w$ equals four. Figure 2 plots $\left|V_{1}(f)\right|^{2}$ for the frequencies up to $f=0.14$. The central peak of the MB taper is more concentrated around $f=0$ than the Slepian taper is. The first sidelobe of the MB taper is visible while the first Slepian sidelobe is much smaller.

Figure 1 plots the logarithm of $\left|V_{1}(f)\right|^{2}$ over the entire frequency range. The MB taper has smaller range bias in the frequency range $|f|<0.3 w$ and in the frequency range $|f|>0.13$. In the middle frequency range, the Slepian taper is clearly better. The Slepian penalty function maximizes the energy inside the frequency band, $[-w, w]$, and thus it is natural that the Slepian tapers do better for $f \sim w$. By using a discontinuous penalty function, the Slepian spectral windows experience Gibbs phenomenon and decay only as $\frac{1}{f},\left(|V(f)|^{2} \sim \frac{1}{f^{2}}\right)$. The MB spectral windows decay as $\frac{1}{f^{2}}$, and thus, it is natural that the MB tapers have lower bias for $f \sim \mathcal{O}(1)$.

Figure 3 plots $\sum_{k=1}^{3}\left|V^{(k)}(f)\right|^{2}$ for $|f|<0.14$ and on this scale, the MB 
tapers are clearly preferable to the Slepian tapers. For larger frequencies, the energy of the multitaper estimate, $\sum_{k=1}^{K}\left|V^{(k)}(f)\right|^{2}$, is very similar to Fig. 2 on the logarithmic scale provided that $K<<N$.

In summary, the sinusoidal tapers perform nearly as well as the MB tapers while the Slepian tapers have several times larger local bias (except when $k \approx 1.2 N w)$. For $k \ll 2 N w$, the Slepian tapers have better broad-band bias protection than the minimum bias tapers do. For $k \sim 2 N w$, the minimum bias tapers provide both smaller local bias and better broad-band protection due to the Gibbs phenomena which the Slepian tapers experience.

\section{Local Error Analysis and Optimal Multita- pering}

We now give a local error analysis of MTSA and determine the optimal number of tapers. Our results are the multitaper analog of the local error analysis of the smoothed periodogram [4, 10]. We assume the time series is a Gaussian processes and do not consider frequencies near $f=0$ and $f=$ $1 / 2$. In this case, the variance of the multitaper estimate is approximately Variance $[\hat{S}(f)] \approx S(f)^{2} \sum_{k=1}^{K} \mu_{k}^{2}$ due to the orthonormality of the tapers

Asymptotically, the local bias of the multitaper estimate of Eq. (3) is

$$
\operatorname{Bias}[\hat{S}]=S(f)\left(\sum_{k=1}^{K} \mu_{k}-1\right)+\frac{1}{2} S^{\prime \prime}(f) \sum_{k=1}^{K} \lambda_{k} \mu_{k},
$$

where $\lambda_{k}=\int_{-1 / 2}^{1 / 2} f^{2}\left|V^{(k)}(f)\right|^{2} d f$. The second term is the MT generalization of (7). When $\sum_{k=1}^{K} \mu_{k} \neq 1$, the MT estimate has bias even in white noise. When we require $\sum_{k=1}^{K} \mu_{k}=1$, the local expected loss simplifies:

Theorem 5.1 For a Gaussian process, away from $f=0$ and $f=1 / 2$, the expected square error of the multitaper spectral estimate (3) with $\sum_{k=1}^{K} \mu_{k}=1$ is asymptotically (to leading order in $K / N$ )

$$
\operatorname{Bias}^{2}+\text { Variance } \approx\left[\frac{1}{2} S^{\prime \prime}(f) \sum_{k=1}^{K} \lambda_{k} \mu_{k}\right]^{2}+S(f)^{2} \sum_{k=1}^{K} \mu_{k}^{2} .
$$

Theorem 5.2 The multitaper estimate which minimizes the local loss (10) (with $\mu_{k} \geq 0$ ) is constructed with the minimum bias tapers. 
Proof: We order the $\mu_{k}$ such that $\mu_{1} \geq \mu_{2} \geq \ldots \geq \mu_{K}$ and define $\mu_{K+1}=$ 0 . Since the weights, $\mu_{k}$ are fixed, we need to minimize $\sum_{k=1}^{K} \mu_{k} \mathbf{u}_{k} \mathbf{A} \mathbf{u}_{k}^{*}$ over all sets of $K$ orthonormal tapers, $\mathbf{u}_{1}, \ldots, \mathbf{u}_{K}$. We split the series in $K$ subseries and minimize each subseries separately:

$$
\begin{aligned}
\min _{\mathbf{u}_{1}, \ldots, \mathbf{u}_{K}} \sum_{k=1}^{K} \mu_{k} \mathbf{u}_{k} \mathbf{A} \mathbf{u}_{k}^{*} & =\min _{\mathbf{u}_{1}, \ldots, \mathbf{u}_{K}} \sum_{k=1}^{K}\left(\mu_{k}-\mu_{k-1}\right)\left(\sum_{j=1}^{k} \mathbf{u}_{j} \mathbf{A} \mathbf{u}_{j}^{*}\right) \\
& \geq \sum_{k=1}^{K}\left(\mu_{k}-\mu_{k-1}\right)\left(\min _{\mathbf{u}_{1}^{(k)}, \ldots, \mathbf{u}_{k}^{(k)}} \sum_{j=1}^{k} \mathbf{u}_{j}^{(k)} \mathbf{A} \mathbf{u}_{j}^{(k) *}\right) \\
& =\sum_{k=1}^{K}\left(\mu_{k}-\mu_{k-1}\right)\left(\sum_{j=1}^{k} \lambda_{A, j}\right)=\sum_{k=1}^{K} \mu_{k} \lambda_{A, j},
\end{aligned}
$$

where the $\lambda_{A, j}$ are the eigenvalues of $\mathbf{A}$, given in increasing order. The $\mathbf{u}_{j}^{(k)}$ are subject to orthonormality constraints that $\mathbf{u}_{j}^{(k)} \cdot \mathbf{u}_{j^{\prime}}^{(k)}=\delta_{j, j^{\prime}}$, but are otherwise independent and minimized separately. In the last line of (11), we use Fan's Theorem [6]: $\min _{\mathbf{u}_{1}^{(k)}, \ldots, \mathbf{u}_{k}^{(k)}} \sum_{j=1}^{k} \mathbf{u}_{j}^{(k)} \mathbf{A} \mathbf{u}_{j}^{(k) *}=\sum_{j=1}^{k} \lambda_{A, j}$, where the $\mathbf{u}_{j}^{(k)}$ are again subject to orthonormality constraints. The theorem is now proved because the MB tapers are precisely the eigenvectors of $\mathbf{A}$.

Theorem 5.3 The uniformly weighted multitaper estimate using $K$ sinusoidal tapers has an asymptotic local loss of

$$
\text { Bias }^{2}+\text { Variance } \simeq\left[\frac{S^{\prime \prime}(f) K^{2}}{24 N^{2}}\right]^{2}+\frac{S(f)^{2}}{K} .
$$

Corollary 5.4 The asymptotic local loss of (12) is minimized when the number of tapers is chosen as

$$
K_{\text {opt }} \sim\left[\frac{12 S(f) N^{2}}{S^{\prime \prime}(f)}\right]^{2 / 5} .
$$

Thus, the optimal number of tapers is proportional to $N^{4 / 5}$ and varies with the ratio of $S(f)$ to $S^{\prime \prime}(f)$. Intuitively 13 shows that fewer tapers should be used when the spectrum varies more rapidly. A key advantage of the MB and sinusoidal tapers is that the tapers need not be recomputed as $K$ is changed. In contrast, the Slepian tapers are most efficient when the bandwidth parameter, $w$, is chosen such that $K \sim 2 N w$. Thus, when the number of tapers is changed, as in (13), the Slepian tapers should be recomputed. 


\section{Smoothed Multitaper Estimates}

In our own comparison of kernel smoothing and multitaper estimation [16], we found that a smoothed multiple taper estimate worked best. We now evaluate the expected error of the kernel smoothed multitaper estimator and show that smoothing the logarithm of the multitaper estimate is useful for estimating the logarithm of the spectrum.

We begin by evaluating that the quadratic estimator (2) which is equivalent to a kernel smoother estimates of the spectrum [4, 10]. Let $\hat{S}(f)$ be the quadratic spectral estimator (2), and smooth it with a kernel $\kappa(\cdot)$ of halfwidth $w$ :

$$
\widehat{\widehat{S}}(f)=\int_{-w}^{w} \kappa\left(\frac{g}{w}\right) \widehat{S}(f+g) d g,
$$

where $w$ is the bandwidth parameter and $\kappa(\cdot)$ is a kernel smoother with domain $[-1,1]$. This can be rewritten as

$$
\widehat{\widehat{S}}(f)=\sum_{n, m=1}^{N} \tilde{q}_{n m} e^{i 2 \pi(m-n) f} x_{n} x_{m},
$$

where $\tilde{q}_{n m}=q_{n m} \hat{\kappa}_{m-n}$ with $\hat{\kappa}_{m}=\int_{-w}^{w} \kappa(g / w) e^{2 \pi i m g} d g$. Thus smoothing replaces the original quadratic estimator with matrix $\left[q_{n m}\right]$ by another quadratic estimator with matrix $\tilde{\mathbf{Q}}=\left[\tilde{q}_{n m}\right]$. By Theorem 5.2, this hybrid method cannot outperform the pure multitaper method with minimum bias tapers.

We now show that combining kernel smoothing with multitapering does improve the estimation of the logarithm of the spectral density, $\theta(f)=$ $\log [S(f)]$. One standard approach is to kernel smooth the logarithm of the tapered periodogram. This approach has the disadvantage that $|y(f)|^{2}$ has a $\chi_{2}^{2}$ distribution and $\log \left[\chi_{2}^{2}\right]$ has a long lower tail of its distribution. As a result, $\log \left[|y(f)|^{2}\right]$ has an appreciable bias and its variance is inflated by $\pi^{2} / 6$. A common alternative is to estimate the spectrum either by kernel smoothing or by multitapering and then to take logarithms. This approach has the disadvantage that the smoothed spectral estimate tends to be more sensitive to nonlocal bias effects than the corresponding smoothed log-spectral estimate.

To robustify the log-spectral estimate while reducing the variance inflation from the long tail, we propose the following hybrid estimate: 1) compute the multitaper estimate using the sinusoidal tapers with $\mu_{k}=\frac{1}{K}$ and then

2) smooth $\hat{\theta}_{M T}(f) \equiv \ln \left[\hat{S}_{M T}(f)\right]-B_{K} / K$, where $B_{K}$ is the bias of $\ln \left[\chi_{2 K}^{2}\right]$. 
$\left(B_{K} \equiv \psi(K)-\ln K\right.$ where $\psi(K)$ is the digamma function). For white noise, the variance of $\hat{\theta}_{M T}(f)=\psi^{\prime}(K) \simeq \frac{1}{K}+\frac{1}{2 K^{2}}$, so the variance enhancement from the logarithm tends rapidly to zero.

In [15], we show that the asymptotic error for this scheme is

$$
\theta^{\prime \prime}(f)^{2}\left[b_{k} w^{2}+\frac{K^{2}}{24 N^{2}}\right]^{2}+\frac{C_{\kappa}}{N w}\left(1+\frac{1}{2 K}\right)^{2},
$$

where $b_{\kappa}$ and $C_{\kappa}$ are constants which depend on the kernel shape. In (16), we assume uniformly weighted sinusoidal tapers are used and $1 \ll K \ll N w$. In (16), one factor of $\left(1+\frac{1}{2 K}\right)$ is the variance enhancement from the logarithmic transformation and one factor of $\left(1+\frac{1}{2 K}\right)$ arises in the variance calculation of (15) with sinusoidal tapers. Optimizing (16) with respect to both $w$ and $K$ yield $w \sim N^{-1 / 5}$ and $K \sim N^{8 / 15}$, thus the smoothing halfwidth $w$ is much larger than $K / N$. The expected error (16) depends weakly on $K$ provided that $1 \ll K \ll N w$.

For simplicity, we set $K=N^{8 / 15}$ and optimize $(16)$ with respect to the halfwidth $w$. The resulting halfwidth depends on $\theta^{\prime \prime}(f): w_{\text {opt }}\left(\theta^{\prime \prime}(f)\right)$ with $w_{\text {opt }} \sim\left|\theta^{\prime \prime}(f)\right|^{-2 / 5} N^{-1 / 5}$. Thus when the log-spectrum varies rapidly, the halfwidth should be reduced as $\left|\theta^{\prime \prime}(f)\right|^{-2 / 5}$.

Since $\theta^{\prime \prime}(f)$ is unknown, we consider two stage estimators which begin by making a preliminary estimate of $\theta^{\prime \prime}(f)$ prior to estimating $\theta(f)$. We then insert the estimate $\theta^{\prime \prime}(f)$ into the expression for $w_{\text {opt }}: w(f)=w_{\text {opt }}\left(\widehat{\theta^{\prime \prime}}(f)\right)$ and use a variable halfwidth kernel smoother with halfwidth $\hat{w}(f)$ to estimate $\theta(f)$. Multiple stage kernel estimators are described in [2, 7, 13, 14, 15]. These multiple stage schemes have a convergence rate of $N^{-4 / 5}$ and have a relative convergence rate of at least $N^{-2 / 9}$. A more detailed description is given in [2, 13, 15].

\section{Application}

We now compare spectral estimates on an actual data series. We use the microwave scattering data set which is described in [16]. The data measures turbulent plasma fluctuations in the Tokamak Fusion Test Reactor at Princeton. The spectrum is dominated by a $1 \mathrm{MHz}$ peak which is quasicoherent. The spectral density varies by over five orders of magnitude. 
The bias versus variance trade-off of Sec. 5 shows that fewer tapers should be used near the peak. To make the spectral estimate smooth, a parabolic weighting of the tapers is used as described in Sec. 3. To determine how many tapers to use locally, we use the multiple stage "plug-in" method as described in the previous section; i.e. we determine the number of tapers using a pre-estimate on the same data. To reduce the fluctuations from the estimate of the optimal number, we use a longer data segment to determine the number of tapers at each frequency. We find the optimal number of sinusoidal tapers is roughly 24 for frequencies in the 200 to $800 \mathrm{kHz}$ range. Near the $1 \mathrm{MHz}$ peak, as few as 12 tapers are used to minimize the local bias error. Between 1300 and $2400 \mathrm{kHz}$, the spectrum is flatter and we use up to 40 tapers.

The dotted line is the sinusoidal multitaper estimate, and the solid, more wiggly, curve is the corresponding Slepian estimate using 24 tapers with $w=60 \mathrm{kHz}$. The $1 \mathrm{MHz}$ peak is poorly resolved in the Slepian estimate, and the regions of high curvature are artificially flattened. For $f \geq 1.5 \mathrm{MHz}$, the Slepian estimate is artificially bumpy due to statistical noise. The variable taper number estimate suppresses these bumps by averaging over a larger frequency halfwidth. We have also used a variable taper number estimate with the Slepian tapers. Since the Slepian parameter, $w$ was fixed at $100 \mathrm{kHz}$ to allow for forty tapers, the artificial broadening was even more exreme.

Comparing with a converged estimate of the spectrum based on $N=$ 45, 000 shows that the sinuosidal taper estimate is more accurate. Another significant difference is that the Slepian multitaper estimate requires much more CPU time than the sinusoidal multitaper estimate.

\section{Conclusion}

We have proposed and analyzed the minimum bias and the sinusoidal tapers, $v_{n}^{(k)}=\sqrt{\frac{2}{N+1}} \sin \frac{\pi k n}{N+1}$, for multitaper spectral estimation. The resulting sinusoidal multitaper spectral estimate is $\hat{S}(f)=\frac{1}{2 K(N+1)} \sum_{j=1}^{K} \mid y\left(f+\frac{j}{2 N+2}\right)-$ $\left.y\left(f-\frac{j}{2 N+2}\right)\right|^{2}$. The sinusoidal tapers have low bias because the frequency sidelobe from $y\left(f+\frac{j}{2 N+2}\right)$ cancels the sidelobe of $y\left(f-\frac{j}{2 N+2}\right)$.

The minimum bias tapers minimize the local bias, $\int_{-1 / 2}^{1 / 2} f^{2}\left|V^{(k)} b(f)\right|^{2} d f$, and have good broad-band bias protection as well. Asymptotically, the quadratic spectral estimate which minimizes the expected local square er- 
ror is a multiple taper estimate using the minimal bias tapers.

The sinusoidal tapers have a simple analytic form and approximate the minimum bias tapers to $\mathcal{O}\left(\frac{1}{N}\right)$. The $k$ th sinusoidal taper has its spectral energy concentrated in the frequency bands $\frac{k-1}{2(N+1)} \leq|f| \leq \frac{k+1}{2(N+1)}$.

The minimum bias and sinusoidal tapers have no auxiliary bandwidth parameter, and the bandwidth of the spectral estimate is determined solely by the number of used tapers. By adaptively adding and deleting tapers, a multitaper estimate with the optimal convergence properties of kernel smoothers can be constructed. In contrast, the Slepian tapers need to be recomputed with a different bandwidth. Thus the Slepian tapers are only practical for fixed bandwidth estimation and this is inherently inefficient.

\section{Appendix: Multitaper decomposition of ker- nel estimates}

In Sec. 6, we showed that kernel smoother estimators (14) have an equivalent multitaper representation (3) We now show that the equivalent multitapers of some popular kernel smoother estimates of the spectrum strongly resemble the $\mathrm{MB} /$ sinusoidal tapers. In one special case, this corresondence is exact; i.e. the smoothed periodogram can be exactly decomposed into MB tapers.

Theorem 9.1 Let $w=\frac{1}{2}$ and $\kappa(f)$ be the parabolic kernel, $\kappa(f)=\frac{3}{2}-6 f^{2}$. The eigenvectors of the kernel smoothed periodogram are exactly the discrete minimum bias tapers.

Proof: The $\tilde{\mathbf{Q}}$ matrix in 15 can be calculated explicitly for this case. We find $\tilde{\mathbf{Q}}=\left[b_{n m}\right]=\frac{1}{N}\left(\frac{3}{2} \mathbf{I}-6 \mathbf{A}\right)$ where $\mathbf{A}$ is the matrix from Lemma 3.2 . Thus $\tilde{\mathbf{Q}}$ and $\mathbf{A}$ have the same eigenvectors.

To illustrate that this result is typical even when we apply a taper and smooth over a small band, we consider a smoothed tapered periodogram with $N=200$. We use Tukey's split-cosine taper [16] and then smooth the estimate with a square box kernel with a halfwidth of .01. We then evaluate the corresponding $\tilde{\mathbf{Q}}$ matrix and compute its eigenvectors. Figure 5 displays the first 4 eigenvectors. They are very close to the sinusoids $\sqrt{\frac{2}{N+1}} \sin \frac{\pi k n}{N+1}$. Table 4 shows that this spectral estimate is virtually a $K=4$ multiple taper 
spectral estimate. After $k>4$, the eigenvalues decrease sharply, and these higher eigenvectors contribute very little to the overall estimate.

\section{References}

[1] M. Amin, "Optimal estimation of evolutionary spectra," I.E.E.E. Trans. on Signal Processing vol. 42, 2?, 1994.

[2] T. Brockman, Th. Gasser and E. Hermann, "Locally adaptive bandwidth choice for kernel regression estimators," J. Amer. Stat. Assoc. 88, 1302-1309 (1994).

[3] T. P. Bronez, Nonparametric Spectral Estimation of Irregularly Sampled Multidimensional Random Processes, PhD Thesis, Arizona State University, 1985.

[4] U. Grenander and M. Rosenblatt, Statistical Analysis of Stationary Time Series, New York: Wiley, 1957.

[5] A. N. Kolmogorov and S. V. Fomin, Reele Funktionen und Funktionalanalysis, Section 7.3.2, Berlin: VEB Deutscher Verlag der Wissenschaften, 1975.

[6] A. W. Marshall and I. Olkin, Inequalities: Theory of Majorization and its Applications, p. 511, New York: Academic Press 1979.

[7] H.-G. Müller and U. Stadtmüller, "Variable bandwidth kernel estimators of regression curves," Annals of Statistics, vol. 15, pp. 182-201, 1987.

[8] C. T. Mullis and L. L. Scharf, in Advances in Spectrum Analysis, S. Haykin, Ed., New York: Prentice-Hall, 1990, Chapter 1, pp. $1-57$.

[9] A. Papoulis, "Minimum bias windows for high resolution spectral estimates," IEEE Trans. Information Theory, vol. 19, pp. 9-12, 1973.

[10] E. Parzens, "On asymptotically efficient consistent estimates of the spectral density of a stationary time series," J. Royal Stat. Soc., vol. 19, pp. 303-322, 1958. 
[11] J. Park, C. R. Lindberg, and F. L. Vernon, "Multitaper Spectral analysis of high frequency seismograms," J. Geophys. Res., vol. 92B, pp. 12765-12684, 1987.

[12] D. Percival and A. Walden, Spectral Analysis for Physical Applications: Multitaper and Conventional Univariate Techniques. Cambridge: Cambridge University Press, 1993.

[13] K. S. Riedel, "Optimal kernel estimation of evolutionary spectra," I.E.E.E. Trans. on Signal Processing vol. 41, 2439-2447, 1993.

[14] K.S. Riedel and A. Sidorenko, "Function estimation using data adaptive kernel smoothers- How much smoothing?" Computers in Physics vol. 8, 402-409, 1994.

[15] K. S. Riedel and A. Sidorenko, "Smoothed log-multitaper spectral estimation and data adaptive implementation," submitted for publication.

[16] K. S. Riedel, A. Sidorenko, and D. J. Thomson, "Spectral density estimation for plasma fluctuations I: Comparison of methods," Physics of Plasmas vol. 1, pp. 485-500.

[17] D. Slepian, "Prolate spheroidal wave functions, Fourier analysis, and uncertainty - V: the discrete case," Bell System Tech. J., vol. 5, pp. 1371-1429, 1978.

[18] D. J. Thomson, "Spectrum estimation and harmonic analysis," Proc. IEEE, vol. 70, pp. 1055-1096, 1982.

[19] D. J. Thomson, "Quadratic inverse spectrum estimates: applications to paleoclimatology," Phil. Trans. R. Soc. Lond. A, vol. 332, p. 539-597, 1990. 


\section{Table Captions:}

Table 1: Convergence of the sinusoidal tapers to the minimum bias tapers. Table 2: Normalized bias term, $4(N+1)^{2} \sum_{k=1}^{K} \int_{-1 / 2}^{1 / 2} f^{2} \sum_{k=1}^{K} f^{2}\left|V^{(k)}(f)\right|^{2} d f$, for $N=50$.

Table 3: Spectral concentration, $\int_{-w}^{w} \sum_{k=1}^{K}\left|V^{(k)}(f)\right|^{2} d f$, for $N=50$.

Table 4: Eigenvectors of the smooth tapered periodogram estimator.

\section{Figure Captions:}

Figure 1: Spectral energy of the minimum bias, sinusoidal and Slepian tapers, $N=200, k=1, w=.01$.

Figure 2: Spectral energy of the minimum bias and sinusoidal tapers, $N=200, k=1$. Figure 3 Spectral energy, $\sum_{k=1}^{3} \int_{-1 / 2}^{1 / 2} f^{2}\left|V^{(k)}(f)\right|^{2} d f$, of the minimum bias and Slepian tapers, $N=200, K=3, w=.01$.

Figure 4: Estimated spectral density of the plasma fluctuations. Dashed line is sinusoidal multitaper estimate and solid line is estimate using Slepian tapers with $w=60 \mathrm{kHz}$. Because the Slepian tapers have a fixed bandwidth, the corresponding estimate spectral density at $1 \mathrm{MHz}$ is artificially broadened while being undersmoothed for $f \geq 1.5 \mathrm{MHz}$.

Figure 6: First eigenvectors of the smooth tapered periodogram estimator. 


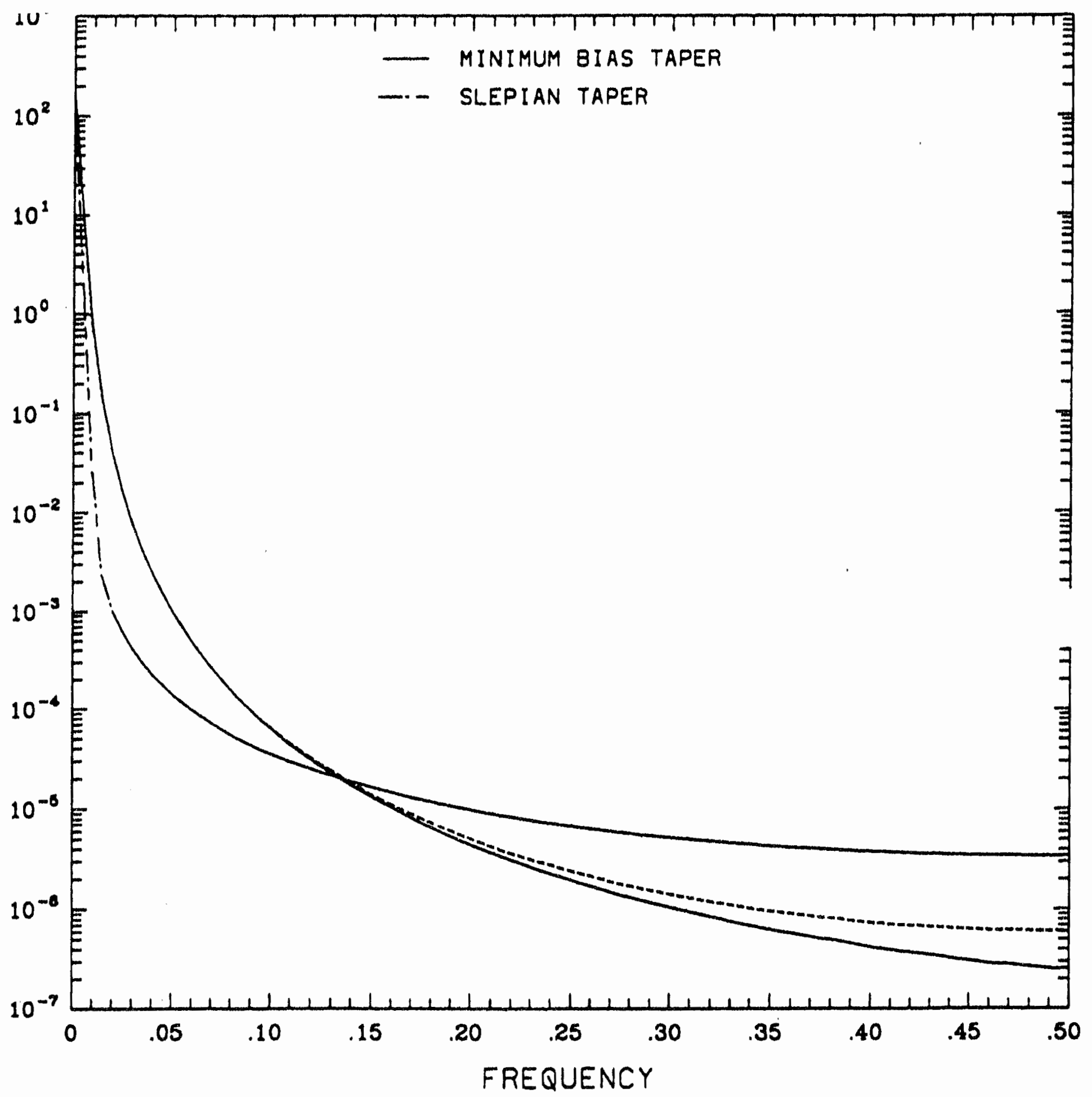

1. Spectral energy of the minimum bias, sinusoidal and Slepian tapers, $=200 \cdot k=1 . *=.01$. 


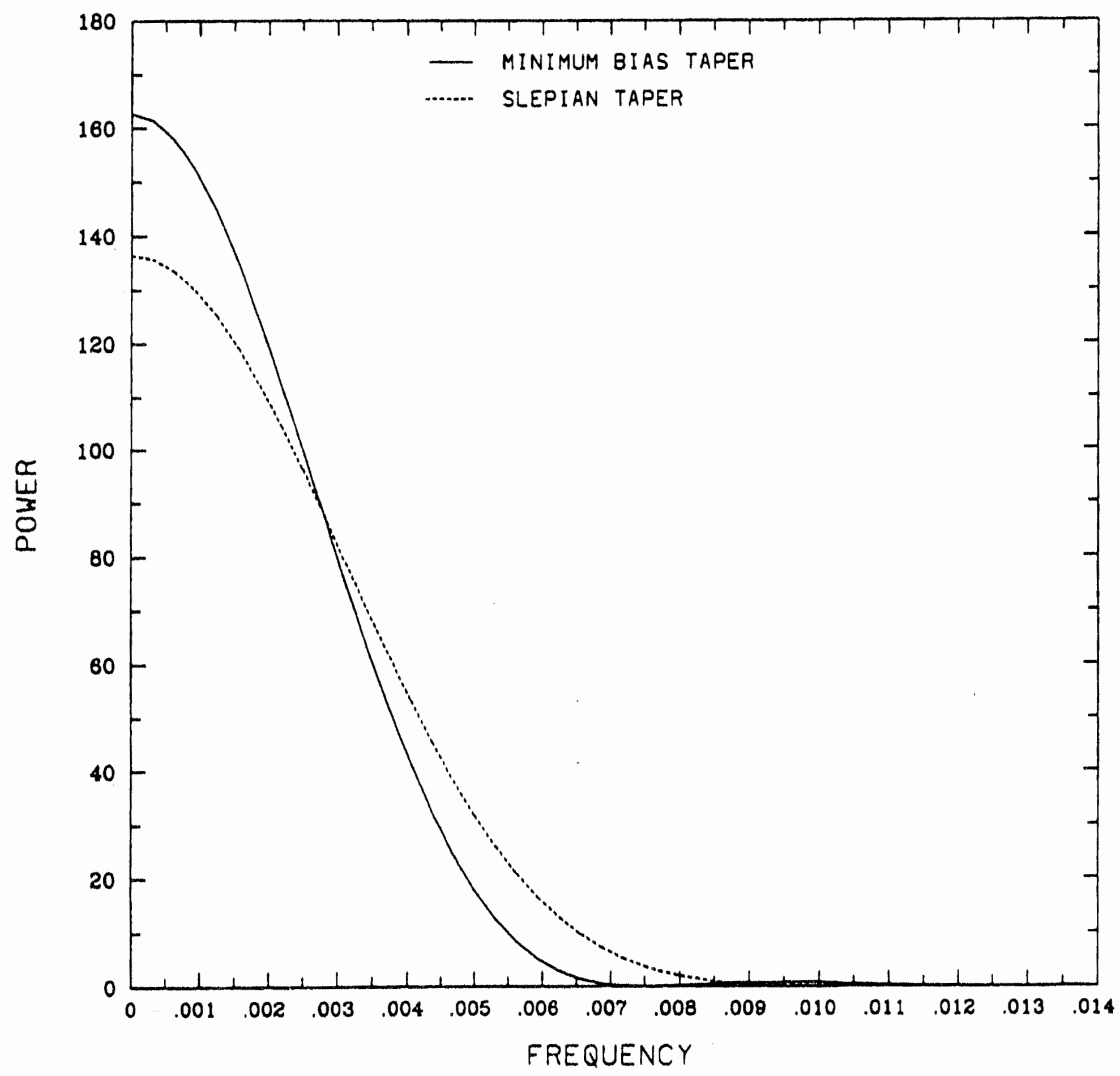

Fig. 2. Spectral energy of the minimum bias and sinusoidal tapers, $V^{*}=200 . k=1$. 


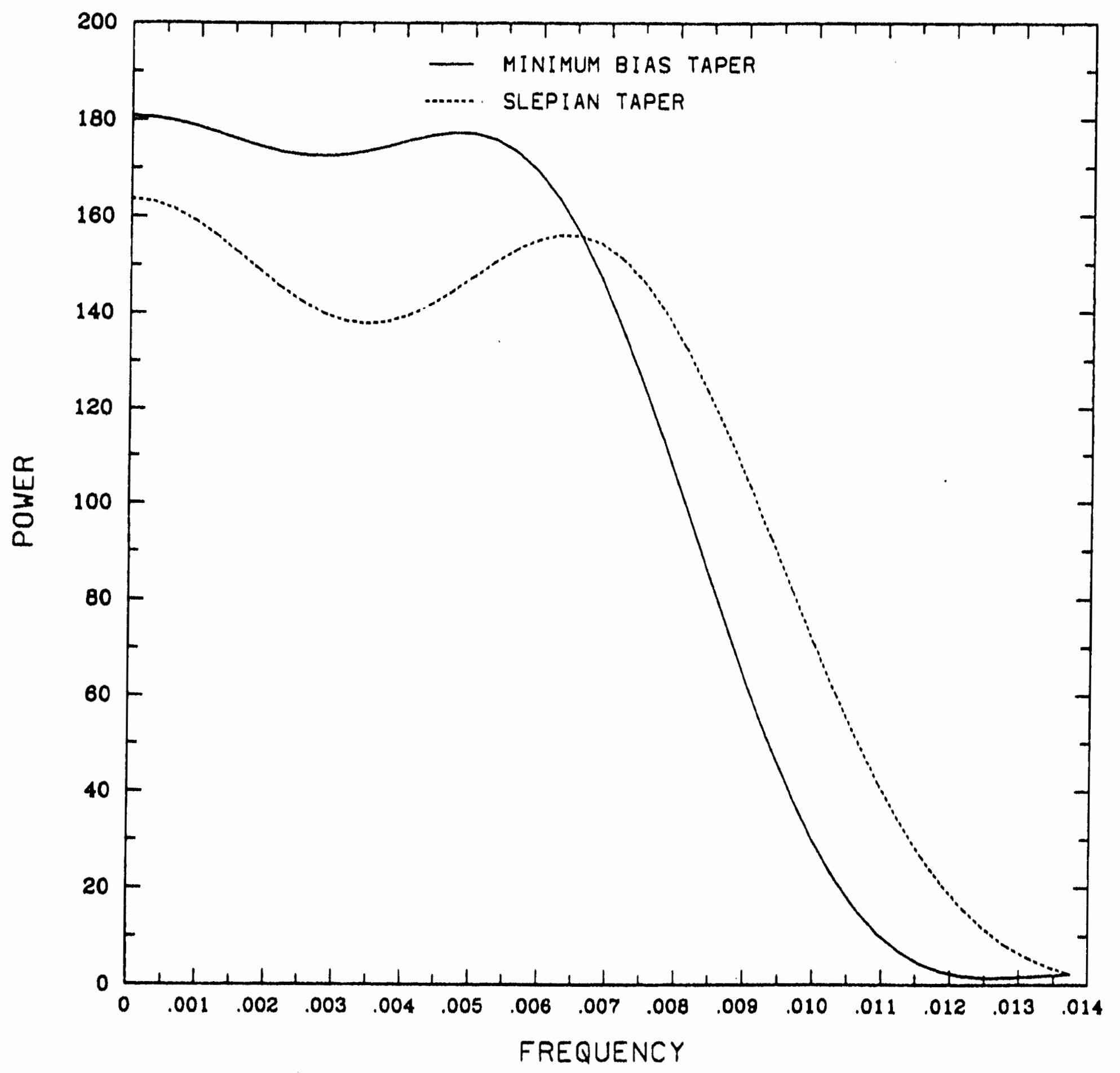

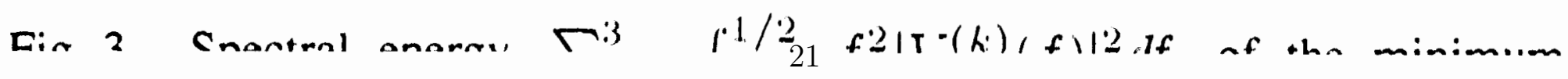




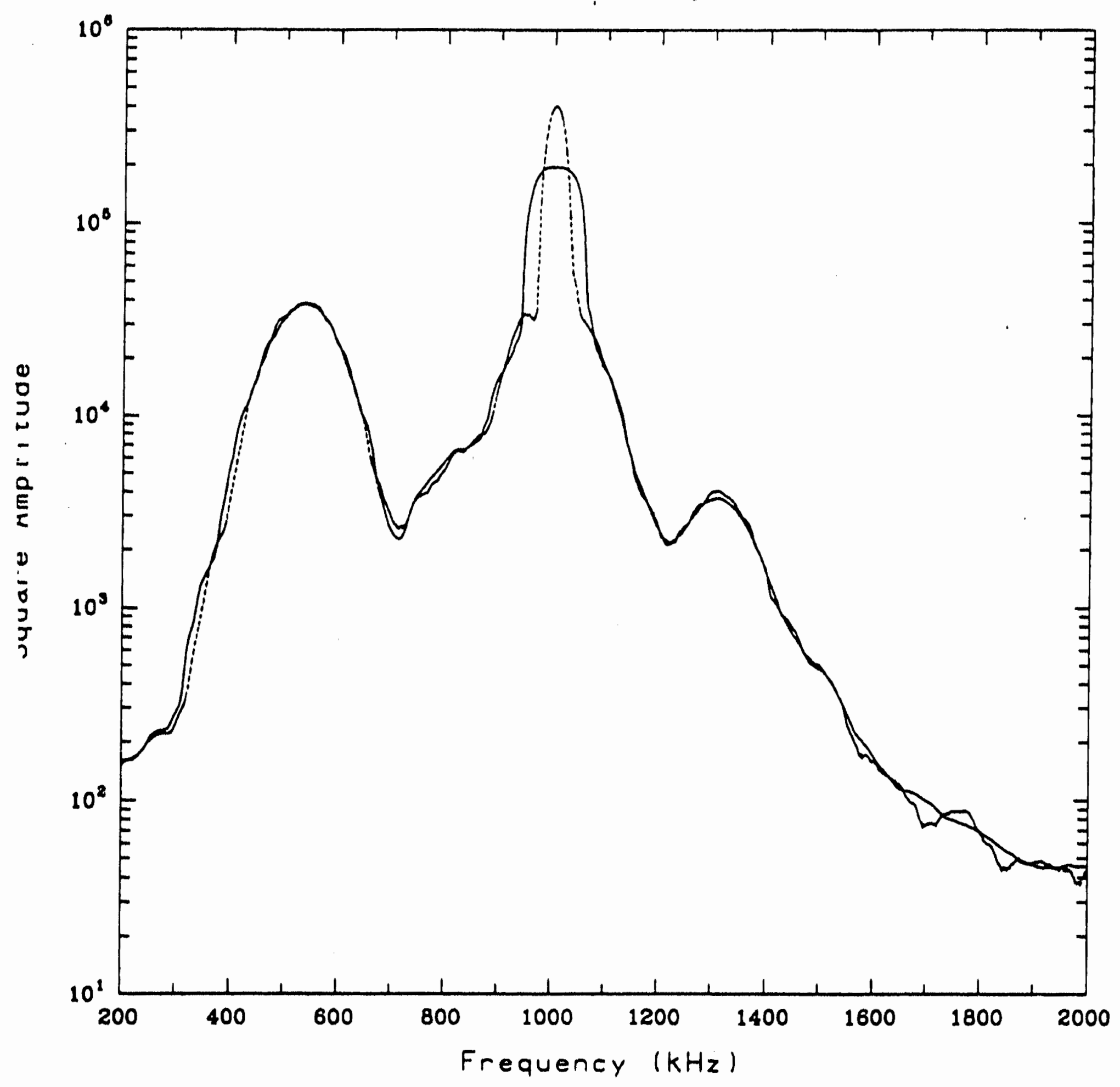

g. 4. Estimated spectral density of the plasma fluctuations. Dashed line is lusoidal multitaper estimate and solid line is estimate using Slepian tapers th $w=60 \mathrm{kHz}$. Because the Slepian tapers have a fixed bandwidth, the rresponding estimate spectral density at $1 \mathrm{MHz}$ is artificially broadened vile being undersmoothed for $f>1$ iMHz 


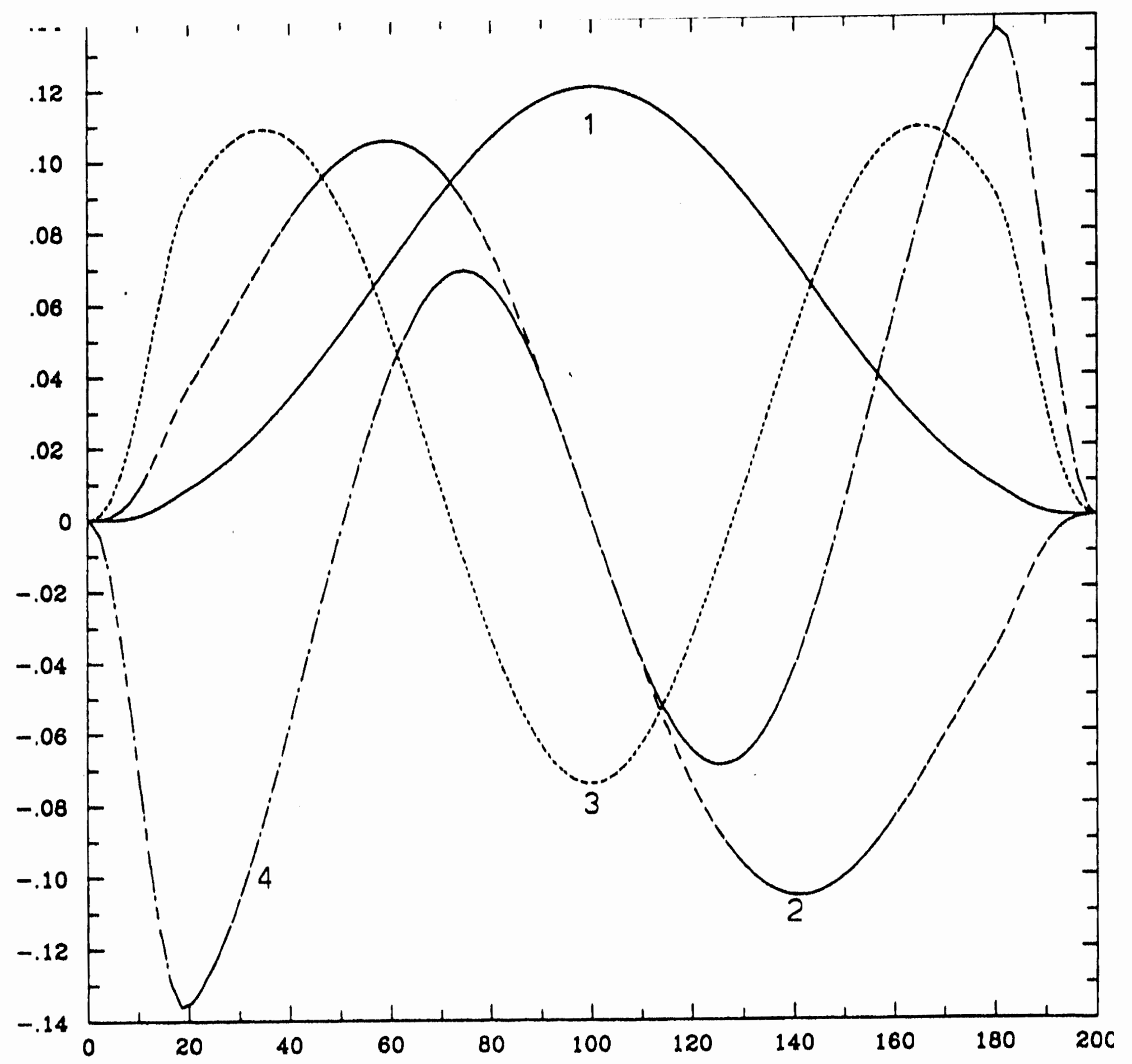

ig. 5. First eigenvectors of the smooth tapered periodogram estimator. 


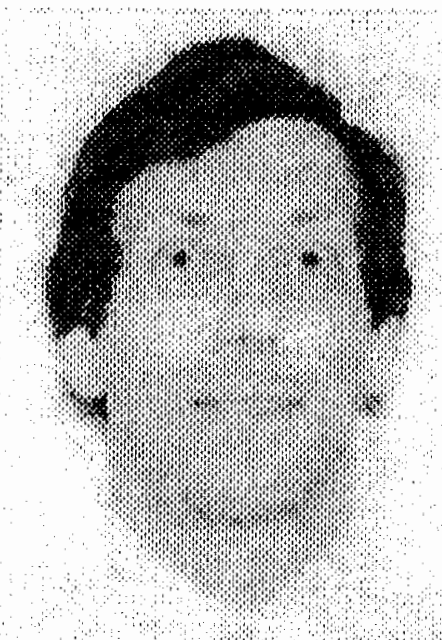

Kurt S. Riedel (M'92) was born in San Bernardino, $\mathrm{CA}$ in 1959. He received the B.A. degree in mathematics and physics with highest honors from the University of California, Berkeley, in 1981, and the M.A. and Ph.D. degrees in applied mathematics from the Courant Institute, New York University, in 1983 and 1985, respectively.

Since 1981 he has been with the Magneto-Fluid Dynamics Division of the Courant Institute, New York University, where he is currently a Research Associate Professor. He has held visiting positions at the Kurchatov Institute and the Max-Planck Institute. He is the author of more than 35 refereed publications and 25 reports and conference proceedings. His early work was in plasma theory. In recent years, he has been involved with the use of advanced signal processing and statistical methods in controlled nuclear fusion research. He has organized two international workshops in this area. His particular research interests are estimation in distributed parameter systems, evolutionary spectral analysis, minimal risk estimation, and structured regression, all with applications to nuclear fusion.

Dr. Riedel is a member of APS. IMS, and SIAM.

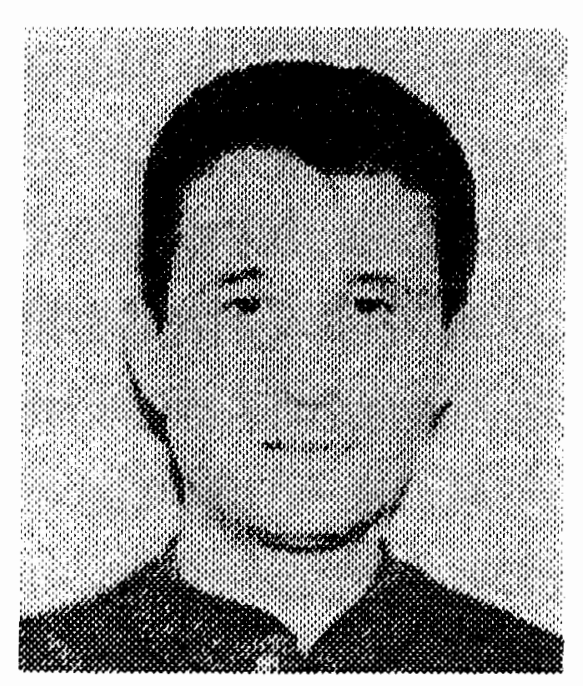

Alexander Sidorenko was born in Simferopol, USSR, in 1954. He received the M.A. degree in applied mathematics from the Moscow Institute of Electronic Engineering in 1976 and the Ph.D. degree in mathematics from Moscow State University in 1982.

From 1982 to 1990, he worked at the National Center for Geophysical Research, Moscow, USSR. Since 1991, he has been at the Courant Institute, New York University, New York, NY. He is the author of more than 50 refereed publications. The areas of his research include discrete mathematics and probability theory. $\mathrm{He}$ is currently concentrating on data-adaptive algorithms for nonparametric function estimation and spectral estimation of digital signals. 
Table 1. Convergence of the sinusoidal tapers to the minimum bias tapers

\begin{tabular}{|r|c|c|}
\hline$N$ & $\max _{k}\left\{\frac{N+2}{k}\left\|\mathbf{v}^{(k)}-\boldsymbol{\nu}^{(k)}\right\|_{L_{2}}\right\}$ & $\max _{k}\left\{\frac{N+2}{k}\left\|\frac{\mathbf{v}^{(k)}}{\left\|\mathbf{v}^{(k)}\right\|_{L_{\infty}}}-\frac{\boldsymbol{\nu}^{(k)}}{\left\|\boldsymbol{\nu}^{(k)}\right\|_{L_{\infty}}}\right\|_{L_{\infty}}\right\}$ \\
\hline 20 & 0.24750 & 0.4602 \\
50 & 0.24844 & 0.4760 \\
200 & 0.24852 & 0.4829 \\
800 & 0.24844 & 0.4844 \\
\hline
\end{tabular}

Table 2. Normalized bias term, $4(N+1)^{2} \sum_{k=1}^{K} \int_{-1 / 2}^{1 / 2} f^{2}\left|V^{(k)}(f)\right|^{2} d f$, for $N=50$

\begin{tabular}{|r|ccccc|}
\hline$K$ & Minimum & Sinusoidal & \multicolumn{3}{c|}{ Slepian tapers } \\
& bias tapers & tapers & $w=0.04$ & $w=0.08$ & $w=0.16$ \\
\hline 1 & 1.0095 & 1.0116 & 1.3439 & 2.6316 & 5.1039 \\
2 & 5.0475 & 5.0580 & 5.7724 & 10.5484 & 20.4670 \\
3 & 14.1328 & 14.1622 & 18.0651 & 23.8086 & 46.1953 \\
4 & 30.2846 & 30.3475 & 58.9520 & 42.5181 & 82.4018 \\
5 & 55.5217 & 55.6366 & 154.4818 & 66.9996 & 129.2087 \\
6 & 91.8634 & 92.0528 & 305.4382 & 99.1800 & 186.7507 \\
7 & 141.3284 & 141.6185 & 496.7959 & 150.0103 & 255.1797 \\
8 & 205.9362 & 206.3570 & 721.1743 & 251.8833 & 334.6717 \\
9 & 287.7056 & 288.2899 & 976.5088 & 437.5993 & 425.4379 \\
10 & 388.6562 & 389.4409 & 1262.4251 & 702.1523 & 527.7433 \\
\hline
\end{tabular}


Table 3. Spectral concentration, $\int_{-w}^{w}|V(f)|^{2} d f$, for $N=50, w=0.08$

\begin{tabular}{|r|c|c|c|}
\hline$k$ & Minimum bias tapers & Sinusoidal tapers & Slepian tapers \\
\hline \hline 1 & .9997 & .9997 & 1. \\
2 & .9988 & .9988 & .9999999 \\
3 & .9972 & .9972 & .9999989 \\
4 & .9940 & .9937 & .99997 \\
5 & .9888 & .9887 & .9995 \\
6 & .9760 & .9753 & .9928 \\
7 & .9381 & .9417 & .9380 \\
8 & .6084 & .6247 & .7002 \\
9 & .1688 & .1780 & .2981 \\
10 & .0637 & .0624 & .0628 \\
\hline
\end{tabular}

Table 4. Eigenvectors of the smooth tapered periodogram estimator

\begin{tabular}{|c|ccc|}
\hline$k$ & $\begin{array}{c}\text { Weight of the eigenvector } \\
\lambda_{k}(B) / \operatorname{tr}(B)\end{array}$ & $\begin{array}{c}\text { Normalized local bias } \\
4(N+1)^{2} \int_{-1 / 2}^{1 / 2} f^{2}|V(f)|^{2} d f\end{array}$ & $\begin{array}{c}\text { Local bias in comparison with } \\
\text { the minimum bias taper (ratio) }\end{array}$ \\
\hline \hline & & & \\
1 & & 1.5138 & 1.509 \\
2 & .2856 & 4.7371 & 1.181 \\
3 & .2828 & 9.6254 & 1.067 \\
4 & .2519 & 19.2095 & 1.198 \\
5 & .1416 & 33.7118 & 1.345 \\
6 & .0340 & 51.3616 & 1.423 \\
7 & .0037 & 72.9747 & 1.486 \\
\hline
\end{tabular}

